\title{
ANÁLISIS Y RECOPILACIÓN DE INFORMACIÓN SECUNDARIA PARA LA ELABORACIÓN DE LA MICROZONIFICACIÓN SÍSMICA PRELIMINAR DE SAN JOSÉ DE CÚCUTA, COLOMBIA
}

Por:

C. H. Flórez Góngora', C. T. Medrano², M. Sarmiento $M^{3}$, C.F.Lozano L. ${ }^{4}$

\section{RESUMEN}

Este proyecto establece una recopilación y análisis básico de la información disponible en lo que respecta a estudios geológicos y geotécnicos desarrollados en un periodo de 20 años (1982-2003) en la ciudad de San José de Cúcuta. Se apoya en la metodología de la Asociación Francesa de Ingeniería Sísmica (por su sigla en francés AFPS, 1995) en lo referente a estudios de Microzonificacion sísmica de Nivel A. La investigación produjo: 1) un mapa digitalizado geotécnico, sustentado en una base de datos que comprende 199 estudios de suelos y 309 excavaciones de un periodo de 20 años y 2) Espectros de respuesta con un amortiguamiento con respecto al critico del $5 \%$ para cuatro perfiles de suelo, con profundidades de $104 \mathrm{~m}, 255 \mathrm{~m}, 192 \mathrm{~m}$ y $83 \mathrm{~m}$ sobre el lineamiento limnologico del Río Pamplonita. Lo anterior constituye una simulación teórica de amplificación por análisis unidimensional utilizando el programa EERA.

Palabras claves: Microzonificacion sísmica, Espectro de respuesta.

\section{ABSTRACT}

This project establishes a compilation and basic analysis of seismic records obtained from geologic and geotechnical studies and collected over 20 years (1982-2003) in different locations of the city of San José de Cúcuta. We follow the procedure manual of the French Association of Seismic Engineering (by its French abbreviation AFPS, 1995) regarding seismic studies of Level A Microzonification (earthquake hazard map). Our results show: 1) a digitized earthquake hazard map based on 199 soil samples analyses and 309 ground excavations performed over a period of 20 years and 2) Response spectrum estimation (for the $5 \%$ damping) for four soil profiles with depths of $104 \mathrm{~m}, 255 \mathrm{~m}, 192 \mathrm{~m}$ and $83 \mathrm{~m}$ on the limnologic line of Pamplonita River. This study is a theoretical simulation of seismic response amplification by a unidimentional analysis using the software EERA.

Key words: Seismic microzonification, Response spectrum.

\section{INTRODUCCIÓN}

La ciudad de Cúcuta, capital del departamento de Norte de Santander, se localiza en la margen occidental del río Pamplonita, a $10 \mathrm{Km}$ de la frontera con la República de Venezuela. Su ubicación corresponde a los $7^{\circ} 54^{\prime}$ de latitud norte y $72^{\circ} 31^{\prime}$ de longitud al oeste de Greenwich. El Valle de Cúcuta se encuentra en un piso térmico cálido, siendo un valle interandino con larga época de sequía (Guhl, 1975), e inmerso en una región definida por una altitud sobre el nivel del mar entre los 100 y los 500 metros, (320 m snm para Cúcuta) y por una temperatura media anual de $27.5^{\circ} \mathrm{C}$ a $29.5^{\circ} \mathrm{C},\left(27.5^{\circ} \mathrm{C}\right.$ para Cúcuta), precipitación media anual $763 \mathrm{~mm}$ y dista de Bogotá $653 \mathrm{Km}$.

La ubicación de San José de Cúcuta sobre una zona de alta sismicidad sobre la falla frontal de la cordillera oriental de Colombia, con una aceleración pico efectiva horizontal de diseño de 0.30g (NSR-98), exige llevar a cabo estudios de rigor sobre los distintos aspectos técnicos y científicos que cubre un estudio completo de Microzonificacion, entendiéndose esta, como el proceso de determinación de la amenaza sísmica, absoluta o relativa, en varios lugares con el fin de delimitar microzonas sísmicas ( AIS, 1996).

\footnotetext{
1 Ingeniero Civil, MSc. Profesor investigador Departamento de Geotecnia y Minería UFPS. Director Grupo GIGA-U.F.P.S. chflorez@bari.ufps.edu.co

2 Ingeniera Civil. Esp. UFPS, Departamento de Matemáticas y Estadística.

3 Ingeniera Civil UFPS, 2004.

4 Estudiante Ingeniería Civil, UFPS. Co-investigador Grupo GIGA.
} 
Análisis y Recopilación de información secundaria para la elaboración de la Microzonificación Sísmica Preliminar de San José de Cúcuta, Colombia

Desde el punto de vista de la actividad sísmica los terremotos más destacados en la región son los ocurridos en los años 1644, 1796, 1875, 1950, 1952, 1980 y 1981. El más intenso ocurrió el 18 de mayo de 1875 causando daños en todas las poblaciones vecinas de Colombia y Venezuela ubicadas a casi $80 \mathrm{Km}$ de radio del área epicentral muy cercana a la ciudad de Cúcuta. Estos antecedentes sísmicos, hacen urgente implementar medidas que les permitan a las autoridades municipales conocer el verdadero nivel de amenaza sísmica en San José de Cúcuta.

\section{METODOLOGÍA}

El desarrollo de este proyecto se apoyó en la metodología de la Asociación Francesa de Ingeniería Sísmica (por su sigla en francés AFPS, 1995) en lo que respecta a estudios de Nivel A, el cual se fundamenta en la recopilación e interpretación de la información disponible.

\section{INFORMACIÓN GEOLÓGICA, DE AMENAZA SÍSMICA REGIONAL Y GEOTECNICA.}

A partir de la obtención y clasificación de estudios geotécnicos y geológicos para distintas zonas de la ciudad se desarrolló una base de datos utilizada para la realización de un mapa geotécnico digitalizado a escala 1:25000. (Ver Anexo No 1). Se presenta a continuación un resumen de los aspectos geológicos, de amenaza sísmica regional y geotécnicos más relevantes de la zona de estudio, constituyéndose un resumen básico de los trabajos de AIS (1996), Inproas Ltda. (1992), e Ingeominas-TNO (1981).

\subsection{Información Geológica.}

San José de Cúcuta se encuentra sobre una estructura geológica binacional, dominada por una serie de fallamientos asociados al sistema de fallas de Boconó y la falla Uribante Caparo del occidente de Venezuela; una actividad tectónica proveniente del nudo sísmico de Bucaramanga al sur-occidente perteneciente a la denominada falla frontal de la cordillera oriental de Colombia.

La geología predominante de la ciudad corresponde a unidades sedimentarias estratificadas y no estratificadas del Terciario y Cuaternario. Al Terciario corresponden las unidades litológicas del grupo Guayabo, formación León y formación Carbonera, caracterizados por una secuencia de ínter estratificación de arcillolitas y de areniscas. Se describen a continuación las unidades predominantes en la zona.

\subsubsection{Terciario-eoceno; Formación Mirador} (Tem). Está compuesta por arenitas de cuarzo, en parte conglomeráticas, con algunas intercalaciones de lodolitas grises. Esta formación, aflora en el flanco Oeste del Anticlinal Tasajero; formando extensos planos estructurales buzantes al Oeste; también aflora en los dos flancos del anticlinal de Cúcuta.

\subsubsection{Terciario-mioceno; Grupo Guayabo} (Tmg). Este grupo esta dividido en dos formaciones:

- Formación Cúcuta. Constituida por lodolitas en capas planas paralelas, con intercalaciones delgadas de arenitas.

- Formación Cornejo. Compuesta por lodolitas alternadas con arenitas y capas ferruginosas.

Aflora entre Cúcuta y Ureña a la margen derecha del Río Pamplonita. También hace parte del flanco Este del Anticlinal Tasajero, conformando el cierre periclinal hacia el Norte. Conforman los anticlinales volcados que separan el Valle de Cúcuta de la Terraza de Juan Atalaya (Comuna 9), así como el sinclinal volcado del Pamplonita cuyo eje pasa próximo a la localidad de San Faustino donde esta cubierto por depósitos de terrazas y aluviones del Río Pamplonita y la Quebrada San Faustino. También aflora por la vía Cúcuta - El Zulia, en la Quebrada la Floresta y Quebrada Seca.

\subsubsection{Terciario-oligoceno; Formación Carbonera} (Tec). Consta de una alternancia de lodolitas grises, sideríticas, con arenitas grises verdosas; hacia la base y parte alta se tienen capas de carbón y estratos 
Análisis y Recopilación de información secundaria para la elaboración de la Microzonificación Sísmica Preliminar de San José de Cúcuta, Colombia

delgados de caliza. Deriva su nombre de la Quebrada Carbonera. Esta formación, aflora en el flanco este del anticlinal de Cúcuta y en el flanco Oeste del Anticlinal de Tasajero, donde forma un plano estructural extenso y prolongado que controla la morfología del área; sobre la vía a Ricaurte, se encuentra expuesta y en contacto fallado con el grupo guayabo.

\subsubsection{Terciario-Oligoceno; formación León} (Tol). Consta de arcillolitas grises verdosas que varían a limolitas hacía la parte superior e inferior. La formación León aflora al Norte y Sur de Cúcuta, conformando el flanco Este del anticlinal de Cúcuta; sobre la margen izquierda del Río Pamplonita desde las inmediaciones de la localidad de la Garita; hasta los alrededores del Grupo Mecanizado Maza №35, al Sur del área urbana de Cúcuta. También se encuentra bien expuesta sobre el flanco Oeste del Anticlinal Tasajero; por la margen derecha que de Cúcuta conduce a Puerto Nuevo, y por la carretera Cúcuta - Villa de Rosario al lado derecho del Río Pamplonita en contacto fallado con rocas del grupo Guayabo. Al Oeste infrayace en contacto discordante el Complejo de limos y cantos rubificados de las terrazas de Juan Atalaya y Belén.

\subsubsection{Depósitos Cuaternarios (Qt, Qal,} Qcr). Depósitos pleistocénicos y más recientes que se distribuyen a lo largo de los valles de los Ríos Pamplonita, Zulia y Táchira; y aquellos que se encuentran en las terrazas levantadas por tectonismo. De los cuales se diferencian los siguientes.

- Qt (Cuaternario de Terraza). Constituido por depósitos antiguos que incluye abanicos aluviales y terrazas abandonadas.

- Qal (Cuaternario Aluvial). Constituido por aluviones recientes formados por los cauces de los ríos mayores.

- Qcr (Cuaternario). Compuesto por un complejo de limos y cantos rubificados.

Estos depósitos están en la planicie del peaje, al frente de San Pedro, donde el Valle se amplía, apareciendo extensos depósitos de terrazas, disectados por la acción dinámica del río, que va estallando gradualmente y dejando acumulados grandes depósitos de material de arrastre en la llanura y lechos actuales. Esta tendencia se mantiene, hasta la confluencia con el Río Táchira al Norte de Cúcuta.

\subsection{Amenaza sísmica regional.}

A continuación se presenta una descripción de dos del más importante sistema de fallas que afectan la zona sobre la cual se emplaza la ciudad de San José de Cúcuta.

\subsubsection{Falla Bocono.}

La falla Bocono se define desde la costa Venezolana hasta la cordillera oriental de Colombia, al sur de Cúcuta, (AIS, 1996). La zona de falla de Boconó es uno de los rasgos geotectónicos mas importantes de la parte noroccidental de América del Sur, se caracteriza morfológicamente por una alineación de valles y depresiones lineales (cuencas de tracción), orientados aproximadamente en dirección N45E. Se extiende por más de $500 \mathrm{Km}$. entre la depresión del Táchira (al sur de Cordero) y el Mar Caribe (área de Morón, Edo.Carabobo), cruzando en forma oblicua a los Andes merideños y cortando el extremo occidental de las Montañas del Caribe (Cordillera de la Costa y Serranía del Interior). A lo largo de toda su extensión, se observan una o varias trazas activas, denominadas falla de Boconó, la cual se caracteriza por escarpas de falla, canales fluviales desplazados, lagunas de falla, lomos de falla y otros rasgos geomorfológicos típicos de fallas rumbo-deslizantes. (PDVSA, 1997), (ROD, 1956a; Alberding, 1957; Von Der Osten y Zozaya, 1959; Bushman, 1959).

\subsubsection{Falla Frontal de la cordillera Oriental.} La Falla Frontal de la Cordillera Oriental presenta una serie de sismos asociados de todo tipo de magnitud y de profundidades focales que llegan hasta unos 50 o $60 \mathrm{Km}$. Conforma uno de los sistemas principales más activos de los Andes del Norte, el cual se prolonga hasta el Golfo de Guayaquil. El tipo de fallamiento, 
Análisis y Recopilación de información secundaria para la elaboración de la Microzonificación Sísmica Preliminar de San José de Cúcuta, Colombia

su longitud y su posición, se combinan con los parámetros de sismicidad para asignarle una magnitud última posible de 8 en la escala Ms. (AIS, 1996, p.20). En el cuadro No 1 se puede observar la magnitud máxima y mínima asociada a cada una de las fuentes sismogemnicas cercana a San José de Cúcuta. Para la Falla Frontal de la Cordillera Oriental, se asocia una magnitud máxima (Mu) de 8.0; una tasa anual de sismos $(\lambda)$ igual 1.16 y una profundidad media de 22 kilómetros, lo cual representa una fuente sismogénica de alta capacidad destructora.

Cuadro No. 1. Parámetros de sismicidad de las fuentes sismogenicas cercanas a San José de Cúcuta.

\begin{tabular}{|c|c|c|c|c|c|}
\hline Fuente & Mo & Mu & $\lambda$ & $\beta$ & $\begin{array}{c}\text { Profundidad } \\
\text { Promedio } \\
(\mathrm{Km})\end{array}$ \\
\hline Boconó & 4.0 & 8.0 & 0.052 & 0.770 & 38 \\
\hline $\begin{array}{c}\text { Buc-Santa } \\
\text { Marta Norte }\end{array}$ & 4.0 & 6.5 & 0.105 & 1.732 & 29 \\
\hline $\begin{array}{c}\text { Buc-Santa } \\
\text { Marta Sur }\end{array}$ & 4.0 & 6.5 & 0.264 & 1.474 & 39 \\
\hline $\begin{array}{c}\text { Frontal } \\
\text { Cordillera } \\
\text { Oriental }\end{array}$ & 4.0 & 8.0 & 1.16 & 0.988 & 22 \\
\hline $\begin{array}{c}\text { Uribante } \\
\text { Caparo }\end{array}$ & 4.0 & 7.0 & 0.079 & 0.758 & 18 \\
\hline
\end{tabular}

Fuente: Adaptada de AIS (1996).

\subsection{Información Geotécnica.}

En esta fase se recolectó información correspondiente a los estudios de suelos existentes en el área de San José de Cúcuta. Para la elaboración de la zonificación geotécnica se consultaron 199 estudios de suelos, repartidos por comunas para facilitar la recopilación y procesamiento de datos. En base a la información en los cuales se presenta una descripción de las características geotécnicas a partir de ensayos de laboratorio; características geológicas y el perfil estratigráfico típico del sector donde se llevo a cabo el estudio de suelos, se produjo el primer mapa digitalizado de zonificacion geotécnica para San José de Cúcuta (Ver Anexo). Este mapa se desarrolló con la colaboración de la Secretaría de Planeación Municipal de Cúcuta y también se encuentra sustentado en los mapas geológicos para la ciudad del Ingeominas y del estudio de Inproas Ltda. (1992).

El análisis de la información recopilada establece que para el periodo de 20 años estudiado, en la ciudad no se han registrado perforaciones para estudios geotécnicos a profundidades mayores a los 5 metros, existiendo escasos estudios con registros de perforaciones mayores a 10 metros y cero estudios para caracterización dinámica de los suelos.

La Gráfica No 1 presenta en porcentaje sobre el total de estudios analizados los estudios geotécnicos llevados a cabo en los últimos 20 años, correspondiendo al año de 1992, el periodo de mayor realización de estudios y con bajas dramáticas para los demás años. Esta información permite inferir el bajo nivel de conocimiento del subsuelo de la ciudad que se ha podido establecer a través de los estudios geotécnicos. La Gráfica No. 2 muestra que en solo tres comunas $(1,2$ y 5 ) de las diez correspondientes al casco urbano de la ciudad, representan espacialmente la mayor concentración de estudios geotécnicos. Lo anterior se explica por la media expansión urbana sufrida sobre los suelos en las zonas, básicamente en construcción de vivienda unifamiliar y edificaciones no mayores de cinco pisos.

A partir de la revisión de los diversos estudios geotécnicos de las firmas de consultoria privada de la ciudad y de tesis de grado sobre geología y geotecnia urbana local, se presenta por comunas las características mas relevantes de los suelos de la ciudad (numerales 3.1.1 a 3.1.10).

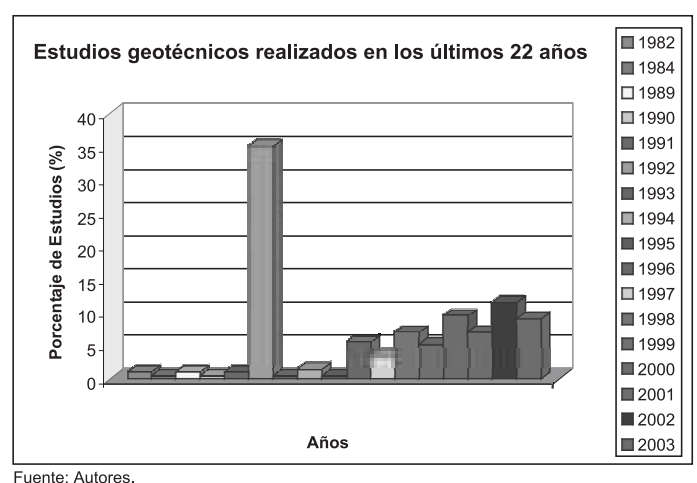

Gráfico No 1. Porcentaje de Estudios geotecnicos realizados por comunas 
Análisis y Recopilación de información secundaria para la elaboración de la Microzonificación Sísmica Preliminar de San José de Cúcuta, Colombia

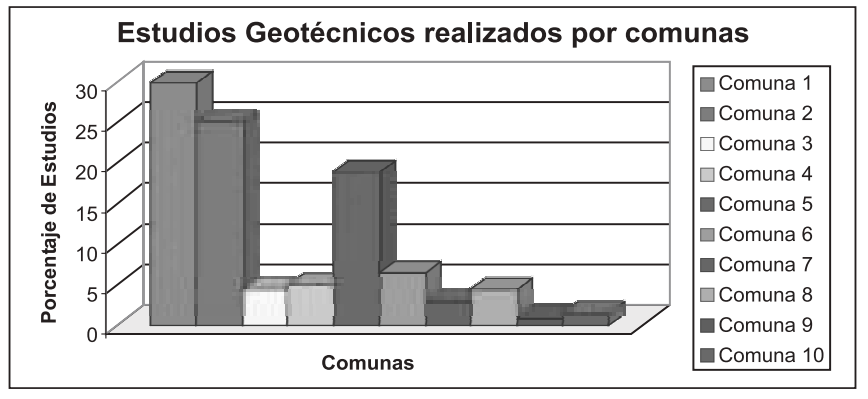

Fuente: Autores.

Gráfico No 2. Estudios geotécnicos realizados por comunas.

\subsubsection{Comuna No 1.}

En la zona predominan, superficialmente materiales de tipo granular y fino. Bajo la superficie se encuentran gravas arcillosas con algo de arena, de color amarillo rojizo, con plasticidad baja a media, se clasifica como GC y GM-GC. Es probable encontrar por debajo de los estratos anteriores un suelo de naturaleza arenoso, denso, de textura granular media a gruesa, se clasifica como una arena algo limosa que presenta ocasionalmente vetas cementadas de arena arcillosa de color gris verdoso y amarillo se clasifica como SM. La plasticidad es baja a nula. Cómo material de soporte aflora mantos aluviales de grava arenosa algo limosa de densidad media.

\subsubsection{Comuna No 2. Sector Centro Oriental.} Los registros estratigráficos hallados confirman la presencia de suelos típicos del cuaternario. El perfil superficial de los suelos está representado predominantemente por series de capas de espesores reducidos compuestas de limos arenosos y en algunos casos, areno limosos ocasionalmente materiales de relleno con predominio de tamaños de arenas y densidades variables. Todas estas capas apoyadas sobre los depósitos fluviales del río Pamplonita caracterizados por gravas, arenas con guijarros y cantos rodados de diferentes diámetros. Las capas de sedimentos que subyacen a los depósitos granulares muestran una litología heterogénea con tendencias hacia los granos y en general exhibiendo densidades bajas. Los espesores de estas capas oscilan entre los $0,0 \mathrm{~m}$ y $2,0 \mathrm{~m}$.

\subsubsection{Comuna No 3. Sector Sur Oriental.} La estratigrafía de la zona esta representada por gravas, guijarros y cantos rodados aglutinados por matrices areno arcillosas precedidos de capas de reducido espesor de sedimentos finos, con alto grado de cementación. Los suelos de esta zona presentan una tendencia creciente de la resistencia con la profundidad. Suelos normalmente consolidados; la expansividad de las capas superficiales es media a baja y un grado de permeabilidad y compresibilidad bajo

\subsubsection{Comuna No 4. Sector Oriental.}

Los suelos predominantes en el área son del tipo residual y corresponden a la meteorización de los depósitos cuaternarios presentes en el área, conformando suelos arcillo arenosos y gravo arcillosos con cantos rodados, estos últimos del complejo de cantos y limos rubificados. El contenido de humedad es relativamente bajo, tendencia creciente con la profundidad. La fracción fina muestra un grado de plasticidad nulo y una resistencia al corte alta. El grado de compresibilidad es bajo y la expansibilidad de las capas superficiales del perfil es muy baja a nula. El grado de permeabilidad puede estimarse como medio a alto para las capas de arena limosa.

\subsubsection{Comuna No 5. Sector Nor Oriental.} Se encuentra caracterizado por terrazas de origen aluvial, pertenecientes a los depósitos cuaternarios que descansan sobre las Formaciones León, Carbonera y Grupo Guayabo. La estratigrafía esta representada por gravas, guijarros y cantos rodados aglutinados por matrices arcillo arenosos de reducido espesor de sedimentos finos; frecuentemente con alto grado de cementación. Predominio de suelos areno limosos y arcillosos o arcillas y limos arenosos de color marrón amarillento y plasticidad media a baja. Los suelos encontrados desmejoran fácilmente sus propiedades de resistencia y deformación al aumentar su humedad.

\subsubsection{Comuna No 6. Sector Norte.}

En el área aflora predominante las formaciones León, formación Carbonera, complejo de Limos y cantos 
Análisis y Recopilación de información secundaria para la elaboración de la Microzonificación Sísmica Preliminar de San José de Cúcuta, Colombia

rubificados y al sur de la formación Guayabo. Se presentan suelos residuales de características predominantemente arcillosa formadas de los procesos de meteorización de las rocas arcillolitas de la formación carbonera; estos suelos son altamente susceptibles a la erosión y presentan un potencial de cambio volumétrico medio a alto y baja permeabilidad.

\subsubsection{Comuna No 7. Sector Nor Occidental.} Según estudios geotécnicos para los perfiles estratigráficos en la zona, se encuentran suelos correspondientes al periodo cuaternario y que constituyen la Formación Guayabo. La zona se caracteriza por presentar mantos intercalados de calizas y arcillolitas. Las arcillolitas son de color amarillo rojizo a amarillo pardo, correspondiéndoles una descripción de roca blanda, la cual se meteoriza fácilmente con la presencia de agua. El perfil estratigráfico típico arroja conglomerado, gravas y guijarros ocasionales, embebidos en matriz arenolimosas; color amarillo; seco superficialmente y humedad creciente con la profundidad; densidad alta a media.

\subsubsection{Comuna No 8. Sector Occidental.}

Desde el punto de vista geológico se presentan en el sector unidades de suelo y unidades de roca; las unidades de suelo están conformadas por suelos residuales provenientes del proceso de meteorización y erosión de rocas arcillosas pertenecientes a la formación León. En la zona predominan suelos de origen residual no transportados (coluviones), estos suelos están conformados por arcillas inorgánicas de plasticidad alta, clasificados como $\mathrm{CH}$ según el sistema unificado de clasificación de suelos y como A-7-6 según el sistema de clasificación AASHTO.

\subsubsection{Comuna No 9. Sector Sur Occidental.} En la zona se presentan depósitos coluvio-aluviales de la edad cuaternaria, producto de avalanchas y flujos de lodos de formaciones que son sedimentarias. Sector intermedio de conos coluvio-aluviales que conformaron el valle donde se construyó Cúcuta. Mantos de limos arenosos, color marrón de gran espesor, al profundizar se encuentra una formación de limos arenosos, color marrón y arcillas arenosas de color amarillo, relativamente blandos.

\subsubsection{Comuna No 10. Sector Sur.}

Deposito cuaternarios y terciarios, las unidades litológicas son complejo de limos y cantos rubificados y formación Guayabo respectivamente. Los perfiles estratigráficos típicos de la zona presentan suelos arcillosos con algo de arena, color marrón en los 0,50m superficiales y habano amarillento y grisáceo a mayor profundidad; secas; plasticidad alta; consistencia muy dura. También se encuentran conglomerado de gravas y guijarros en matriz de arenas arcillosas, color habano amarillento, secas, plasticidad media y densidad alta.

4. Análisis de estratigrafías para modelamiento dinámico.

A partir de la obtención de cuatro registros de perforaciones profundas $(104 \mathrm{~m}, 255 \mathrm{~m}, 192 \mathrm{~m}$ y $83 \mathrm{~m}$ ) ejecutados por el Ingeominas y TNO para el estudio de abastecimiento de agua de la ciudad de Cúcuta en el año de 1981 sobre el lineamiento del río Pamplonita, se estableció un modelo geotécnico al cual se le calcularon de manera teórica sus propiedades dinámicas utilizando los modelos hiperbólicos de comportamiento dinámico de suelos de (Broker y Irebend (1965), Hardin y Black (1968), y Hardin y Drnevich (1972). En el anexo No 2 se encuentra el cuadro de propiedades dinámicas de los perfiles analizados, que corresponden a unidades litológicas del valle aluvial del Río Pamplonita (Sectores Oriental, Sur Oriental). El análisis permitió obtener información del comportamiento local para los cuatro perfiles de suelos de la ciudad (efecto de sitio) ante la eventualidad de ser sometidos a tres señales de fuentes sísmicas diferentes, utilizando el sismo de Loma Prieta y dos sismos del nido de Bucaramanga. La información básica de los mismos se establece en el cuadro № 2 . y a continuación sus registros acelerográficos. 
Análisis y Recopilación de información secundaria para la elaboración de la Microzonificación Sísmica Preliminar de San José de Cúcuta, Colombia

Cuadro No. 2. Información de las Señales sísmicas utilizadas para el cálculo de espectros de respuesta.

\begin{tabular}{|c|c|c|c|c|c|c|c|c|}
\hline Sismo & Fecha & \multicolumn{2}{|c|}{ Epicentro } & Prof.(km) & Ms & Estación & \multicolumn{2}{|c|}{$\begin{array}{c}\text { Ubicación } \\
\text { Estación }\end{array}$} \\
\hline & $\begin{array}{c}\text { Latitud } \\
\text { Norte }\end{array}$ & $\begin{array}{c}\text { Longitud } \\
\text { Oeste }\end{array}$ & & & & $\begin{array}{c}\text { Latitud } \\
\text { Norte }\end{array}$ & $\begin{array}{c}\text { Longitud } \\
\text { Oeste }\end{array}$ \\
\hline $\begin{array}{c}\text { Nido de } \\
\text { B/manga }\end{array}$ & $14 / 04 / 99$ & $6.820^{\circ}$ & $73.140^{\circ}$ & 170 & 6.1 & $\begin{array}{c}\text { Cúcuta } \\
\text { (CCUC1) }\end{array}$ & $7.878^{\circ}$ & $72.509^{\circ}$ \\
\hline $\begin{array}{c}\text { Nido de } \\
\text { B/manga }\end{array}$ & $08 / 11 / 99$ & 6.90 & $-73.18^{\circ}$ & 160 & 6.5 & $\begin{array}{c}\text { Ocaña } \\
\text { (CCONA) }\end{array}$ & $8.20^{\circ}$ & $-73.40^{\circ}$ \\
\hline Loma Prieta & $17 / 10 / 89$ & - & - & 19 & 7.1 & $\begin{array}{c}\text { Diamond } \\
\text { Hts } \\
\text { (USA) }\end{array}$ & - & - \\
\hline
\end{tabular}

\subsection{Registros acelerográficos.}

En las gráficas No 3, 4 y 5 se muestran los registros acelerográficos de las señales utilizadas para obtención de espectros de respuesta. La primera correspondiente al sismo de Loma Prieta, seguidamente dos sismos originados en el Nido de Bucaramanga.

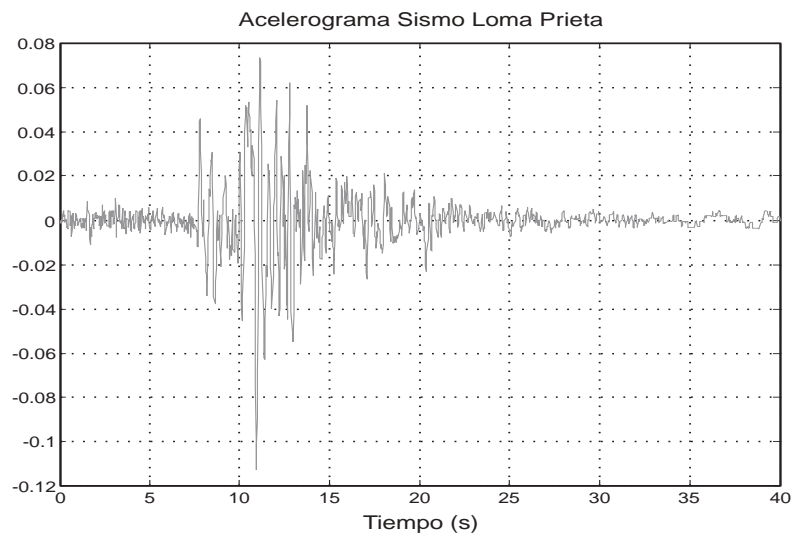

Gráfico No 3. Acelerograma Sismo Loma Prieta

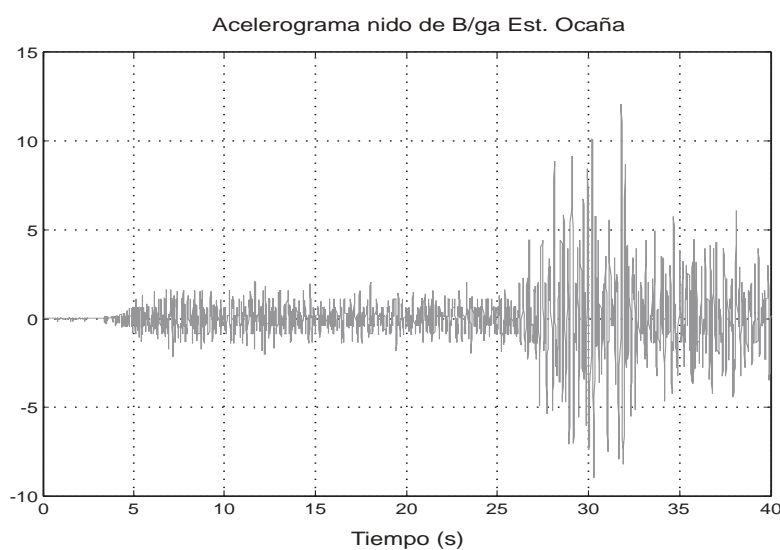

Gráfico No 4. Acelerograma Sismo Nido de Bucaramanga Estación Cúcuta

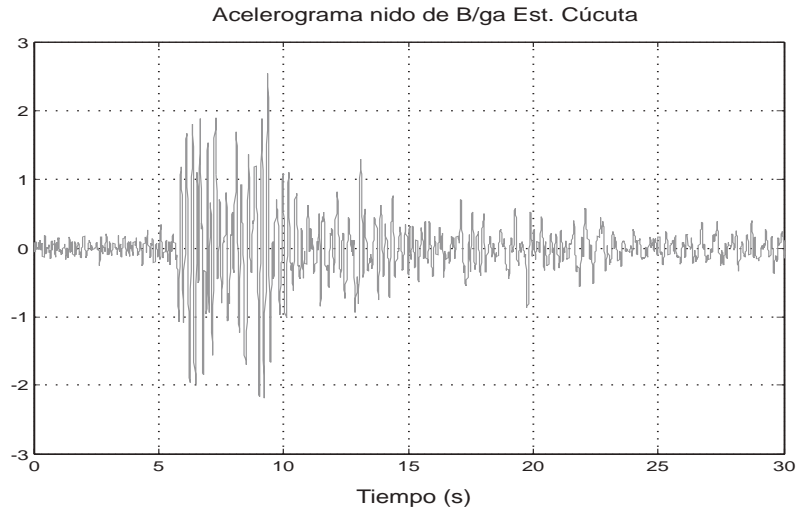

Gráfico No 5. Acelerograma Sismo Nido de Bucaramanga Estación Ocaña

5. Resultado teórico de amplificación por análisis unidimensional utilizando el programa EERA. De las graficas de espectros de respuesta (Gráficos No 6,7, 8 y 9) para los perfiles de suelo analizados con un $5 \%$ de amortiguamiento con respecto al critico, se establece que los periodos ( $T$ ) dominantes de vibración de los depósitos, puede resumirse así:

Perfil 1, 0, $1<\mathrm{T}<0,5$; perfil 2, $\mathrm{T}=0,5$; perfil 3 con un periodo $T$ de 0,3 segundos y perfil $4,0,2<T<0,9$ segundos.

Lo anterior establece periodos dominantes bajos y una mayor amplificación de la señal para el sismo del nido de Bucaramanga captado en la estación Cúcuta.

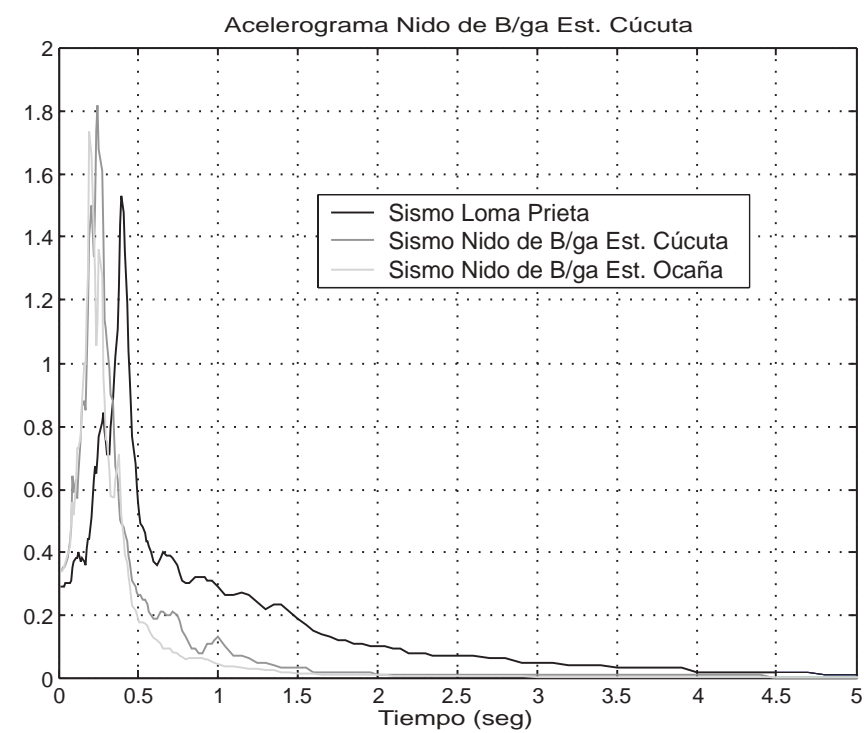

Gráfico No 6. Espectros de Respuesta para el Perfil 1. 
Análisis y Recopilación de información secundaria para la elaboración de la Microzonificación Sísmica Preliminar de San José de Cúcuta, Colombia

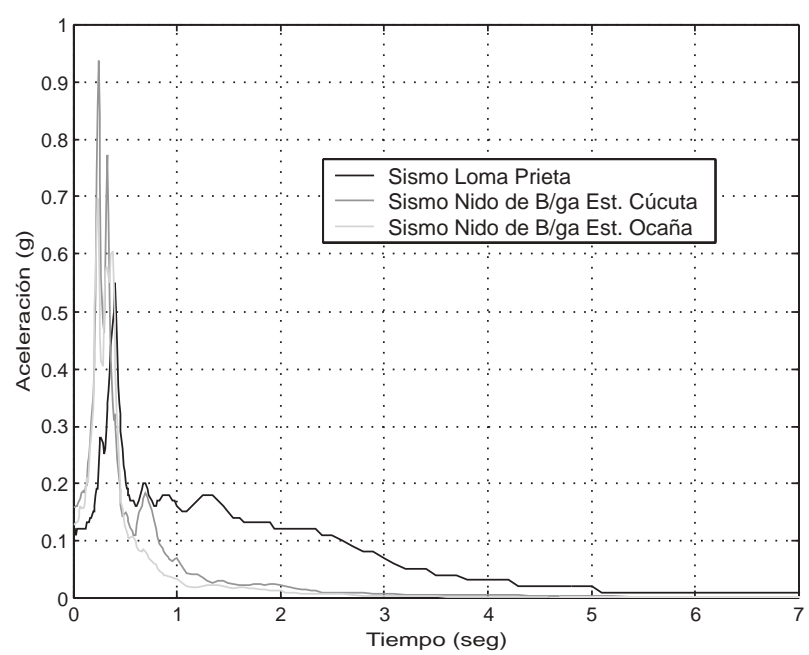

Gráfico No 7. Espectros de Respuesta para el Perfil 2

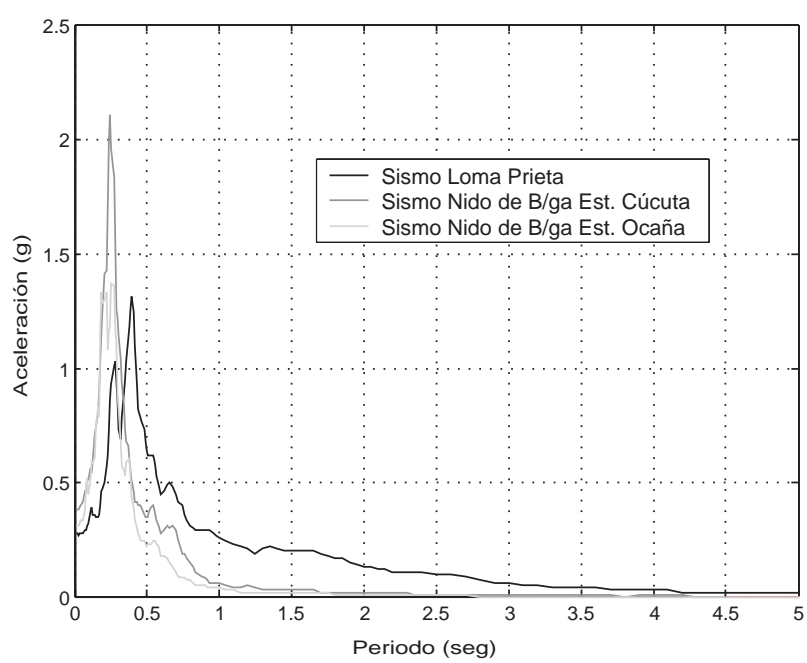

Gráfico No 8. Espectros de Respuesta para el Perfil 3

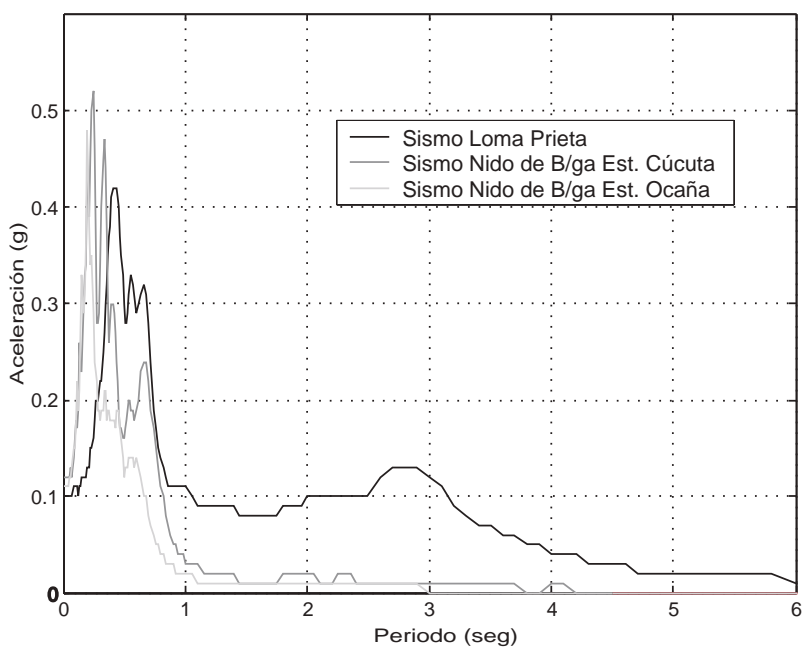

Gráfico No 9. Espectros de Respuesta para el Perfil 4.

\section{CONCLUSIONES}

1. La información obtenida permitió realizar el primer mapa digitalizado de zonificacion geotécnica de la ciudad de Cúcuta, el cual deberá mejorarse con los estudios que para tal fin se ejecuten en un proyecto de Microzonificacion sísmica. Se requiere una mayor información de las estratigrafías para las distintas unidades litológicas dominantes en la ciudad, por cuanto los estudios de suelos realizados hasta el momento cubren los primeros tres metros de profundidad, medida que se encuentra por debajo de la profundidad mínima exigida en estudios geotécnicos, según Titulo H de la NSR-98.

2. Los aspectos de sismicidad histórica e instrumental no se tuvieron en cuenta para el presente análisis por cuanto la información obtenida a nivel local es irrelevante frente al Estudio General de Amenza Sísmica de Colombia, AIS(1996), Ingeominas (1998).

3. De acuerdo al estudio teórico de amplificación por análisis unidimensional utilizando el programa EERA, se puede inferir:

3.1. Los espectros de respuesta muestran amplificación en la mayoría de los perfiles y probados para las tres señales, en periodos bajos $(0,1<T<1,0$ s). Esto podría indicarnos que en la eventualidad de ocurrencia de un sismo con magnitudes comprendidas entre 6.5 y 7.1. Las estructuras que sufrirían mayor impacto serían aquellas estructuras de bajos periodos, tales como edificaciones pequeñas de vivienda unifamiliar y edificios menores a 5 pisos. Este aspecto deberá corroborarse en la ejecución de la micro zonificación sísmica, por cuanto los resultados obtenidos son producto de una simulación teórica a partir de datos existentes y de supuestos sobre la teoría desarrollada hasta el momento para el cálculo de las propiedades dinámicas de depósitos de suelos (arenas y arcillas).

3.2. Los perfiles estratigráficos presentan las siguientes rangos de aceleraciones espectrales: perfil 1: 
Análisis y Recopilación de información secundaria para la elaboración de la Microzonificación Sísmica Preliminar de San José de Cúcuta, Colombia

$1,5 \mathrm{~g}<\mathrm{a}<1,8 \mathrm{~g}$; perfil 2:0,55g<a<0,9g; perfil $3: 1,3 \mathrm{~g}<\mathrm{a}<2,2 \mathrm{~g}$ y perfil $4: 0,42 \mathrm{~g}<\mathrm{a}<0,52 \mathrm{~g}$. Lo anterior indica que los depósitos similares a los del perfil 3 presentarían la mayor aceleración espectral, $2,2 \mathrm{~g}$. Sin embargo, puede concluirse que dada la similitud de los perfiles, por encontrarse sobre la misma unidad litológica, y solo variando la profundidad, la respuesta de los depósitos son similares, dado que no existe variaciones abruptas que pudieran inferir una deficiencia en la información o la existencia de factores no cabalmente comprendidos en este tema. (Sarria, 1995).

4. Las conclusiones anteriores deben guiarnos hacia una investigación completa de los aspectos dinámicos de los depósitos de suelos de la ciudad para orientar los sistemas de construcción ajustados a las especificaciones técnicas del uso del suelo y cumplir con la reglamentación de la Norma Sismo Resistente, la cual se centra en la prevención de catástrofes y perdidas humanas por causa de terremotos.

5. La micro zonificación deberá orientar trabajos en distintas áreas: recopilación de la información sobre el riesgo sísmico; historia sísmica y sismicidad actual; geología y tectónica (causa de los sismos); paleosismología; conocimiento de las propiedades dinámicas de los suelos de Cúcuta; amenaza sísmica (estadística).; censo de las construcciones; digitalización del plano base de San José de Cúcuta.; simulación numérica de terremotos y el análisis de señales de fuentes sismogénicas de campo cercano.

\section{REFERENCIA BIBLIOGRÁFICA}

Asociación de Ingeniería Sísmica, Comité AIS 300. Estudio General de Amenaza Sísmica de Colombia, Octubre 1996.

Association Française du Génie Parasismique-. Guidelines for microzonation studies. Paris: AFPS. 1995.1p.

Braja, M Das. Pprinciples of Soil Dynamics. California: State University, Brooks/Cole 1993. 159 p.

CEDERI. Microzonificacion Sísmica y Estudios de Vulnerabilidad y Riesgo. Centro de investigación en materiales y obras civiles. Bogotá: Universidad de los Andes, 2003.108 p.

Guhl, Ernesto. Colombia: Bosquejo de su geografía tropical. Tomo 1. Instituto Colombiano de Cultura, 1975.

Ingeominas-TNO. "Estudio Hidrogeológico para el Abastecimiento de Agua en la zona de Cúcuta". Cúcuta: 1981.

Ingeominas-Universidad de los Andes, Micro zonificación Sísmica de Bogotá, Agosto 1997.

Inproas Ltda. Estudio Preliminar de zonificacion de áreas según la aptitud del uso del suelo en San José de Cúcuta, 1992.

Ministerio de Ciencias y Tecnología. III Coloquio sobre Microzonificación sísmica, del 15 al 18 de julio del 2002, Fundación Venezolana de Investigación Sismológicas, Venezuela: Ministerio de Ciencias y Tecnología, 2003.

Norma Colombiana Sismo Resistente (NSR 98), Decreto Ley 400 de 1997.

Rodríguez G, Edgar E. Proyecto de Microzonificación Sísmica del Área Metropolitana de Cúcuta (Norte de Santander), Colombia. Cúcuta: Ingeominas. 1994. $257 \mathrm{p}$.

Sarriá Molina, Alberto. Ingeniería Sísmica. Ediciones Uniandes. Bogotá: 1995. 164 p.

Sarriá Molina, Alberto. Métodos geofísicos con aplicaciones a la ingeniería Civil. Bogotá: Uniandes, 1996. $145 \mathrm{p}$.

Villamizar Laguado, William y Rodríguez Sarmiento, Jesús Alfonso. Tentativa de Evaluación del Riesgo Sísmico para el Norte de Santander, Obtención de Parámetros Sísmicos. Cúcuta: U.F.P.S., 1991.258 p.

\section{ANEXOS}

Anexo 1. Mapa de Zonificacion Geotecnica de Cúcuta. Anexo 2. Cuadro de parámetros dinámicos para los perfiles analizados.

Fecha recibido: Octubre 20 de 2005

Fecha aceptación: Noviembre 30 de 2005 
Análisis y Recopilación de información secundaria para la elaboración de la Microzonificación Sísmica Preliminar de San José de Cúcuta, Colombia

Anexo 1. Mapa digitalizado de la Zonificación Geotécnica de San José de Cúcuta.

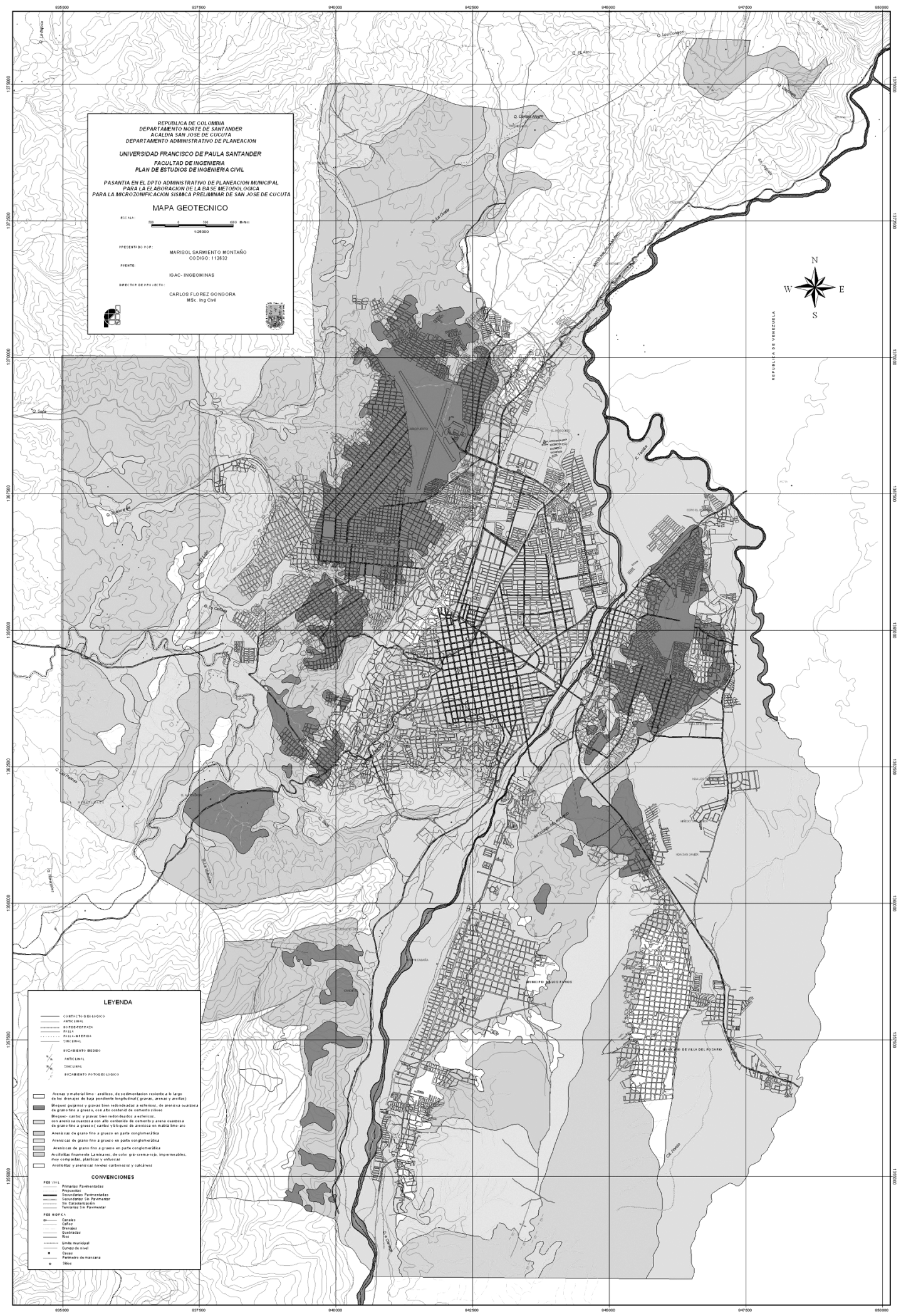


Análisis y Recopilación de información secundaria para la elaboración de la Microzonificación Sísmica Preliminar de San José de Cúcuta, Colombia.

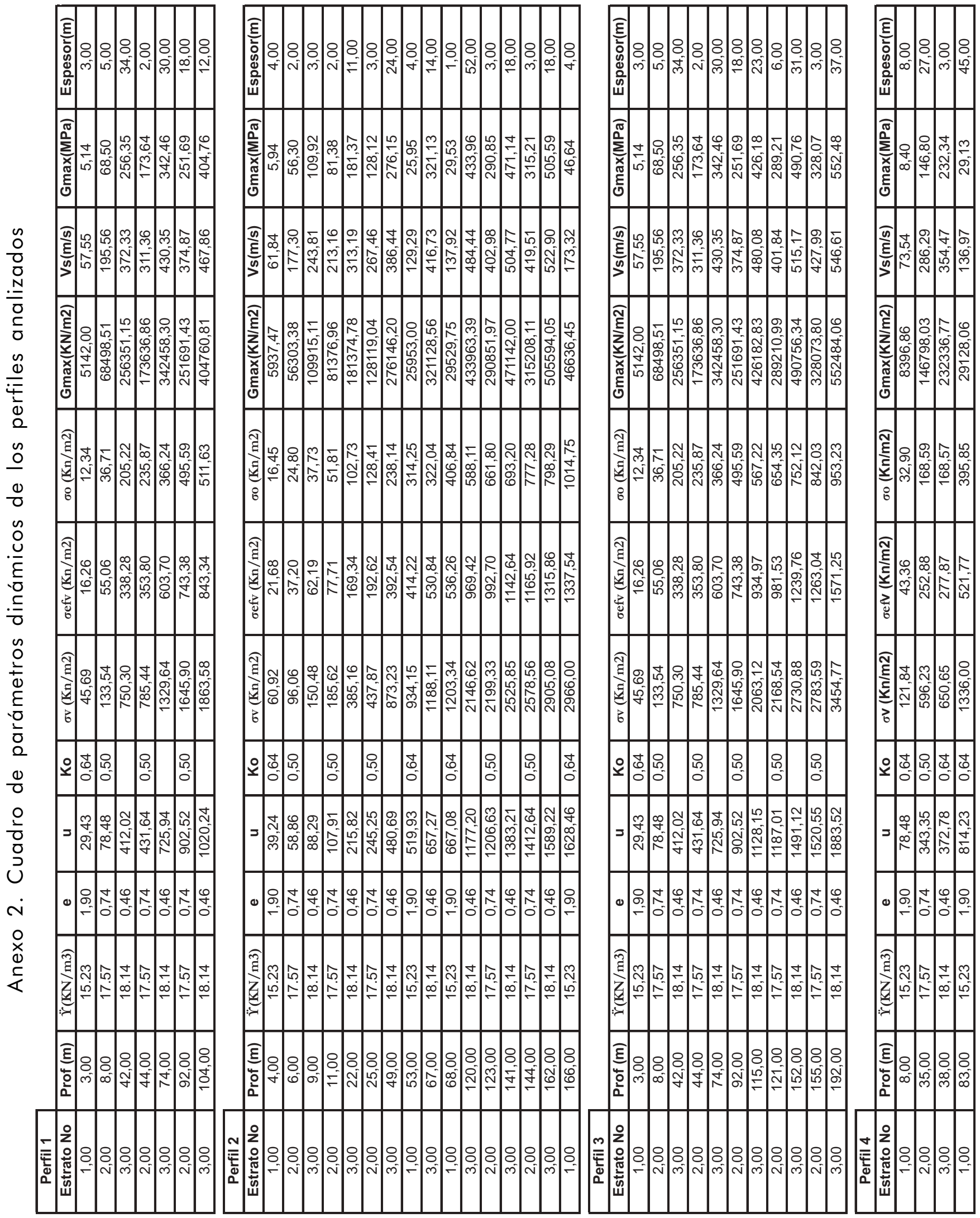

